\title{
Investigation of the behavior of protection elements against field radiated line coupled UWB-pulses
}

\author{
R. Krzikalla and J. L. ter Haseborg \\ Institute of Measurement Technology and Electromagnetic Compatibility, Hamburg, Germany
}

\begin{abstract}
To protect electronic systems against electromagnetic interferences in general nonlinear protection circuits are used. These protection circuits are optimized mostly against special transient interferences such as lightning electromagnetic pulses (LEMP) or electromagnetic pulses caused by nuclear explosions (NEMP). Previous investigations have shown that these protection elements could be undermined by so-called ultra wideband (UWB) pulses. Thereby a direct charge of the UWB-pulse to the elements has been assumed. This assumption was a worst case approximation because in practice UWB-pulses only get into systems by coupling effects. In this investigation the behavior of typical nonlinear protection elements has been tested with field radiated line coupled UWB-pulses. For that line coupled UWB-pulses have been defined depending on the coupling behavior of typical electronic systems and a possibility of generation of this kind of pulses is presented. After it typical nonlinear protection elements such as spark gaps, varistors and protection diodes have been tested with the previously defined test pulses. Finally the measured behavior of the elements has been compared with the behavior by direct charged UWBpulses and the protection effect of the elements against field radiated line coupled UWB-pulses is re-evaluated.
\end{abstract}

\section{Introduction}

The protection of electronic systems against external electromagnetic interferences is very important for an undisturbed functionality of the system. Especially in security relevant systems the requirements for a sufficient protection will be clear because in worst case a system breakdown would be very expensive or could threaten human life. There are sufficient protection circuits available which protect sys-

Correspondence to: R. Krzikalla

(krzikalla@tu-harburg.de) tems against natural interferences, such as lightning electromagnetic pulses (LEMP) or electrostatic discharges (ESD), or against man-made noise just like electromagnetic pulses caused by nuclear explosions (NEMP). In current investigations high amplitude signals with an extremely broad frequency spectrum became more and more important. These so-called ultra wideband (UWB) pulses can be described in time domain by a double exponential time characteristic with a rise time shorter than $150 \mathrm{ps}$ and a pulse width of a few nanoseconds. Previous investigations have been demonstrated that first these kinds of extremely fast transients have destructive effects on electronic systems and second they could undermine traditional nonlinear protection elements (Krzikalla et al., 2003). In that case the behavior of traditional protection elements such as spark gaps, varistors and protection diodes have been determined by using directly the double exponential UWB-pulses. But this assumption is only a worst case approximation to get an idea of the behavior of the elements in a first approach. In practice the disturbance of a system is mostly caused by field radiated interferences which couples into the system (Sabath et al., 2002). Therefore in this investigation the behavior of protection elements has been described by impact with line coupled interferences caused by field radiated UWB-pulses. For this different test pulses have been defined which occur due to the coupling behavior of typical electronic systems. With these test pulses the response behavior of traditional protection elements has been determined which are often used in protection circuit against electromagnetic interferences such as LEMPs of NEMPs. But also new protection elements have been tested which can be used directly against double exponential UWB-pulses (Krzikalla et al., 2003). The results of the measured response behavior have been compared and evaluated with the behavior by impact of direct double exponential UWB-pulses. 


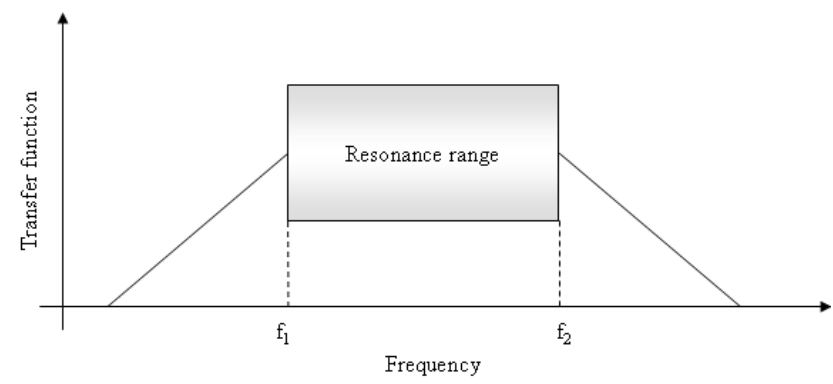

Fig. 1. General coupling behavior of a dipole structure.

\section{Definition and generation of the test pulses}

As mentioned before the disturbing interferences result from coupling effects of field radiated UWB-pulses into the system. To determine the time characteristic of the resulting interference analytically the transfer function of the system has to be known. As described by Nitsch (2005) the transfer function of a system with only a few different coupling paths can be very extensive. For large complex systems such as steering-, control- or computer-systems a transfer function only can be approximated. But this approximation can be done with the fact that the transfer function can be modeled by the transfer behavior of a dipole structure (Nitsch, 2005). The general transfer characteristic of a dipole structure is depicted in Fig. 1 (Baum, 1992). The characteristic corner frequencies $f_{1}$ and $f_{2}$ can be calculated with Eqs. (1) and (2) and they are related to the minimal and maximal coupling path lengths of the system $l_{\min }$ and $l_{\max }$ :

$f_{1}=\left(\frac{3}{4}\right) \cdot \frac{c}{4 \cdot l_{\max } \cdot \sqrt{\varepsilon_{r} \mu_{r}}}$
$f_{2}=\left(\frac{3}{2}\right) \cdot \frac{c}{2 \cdot l_{\min } \cdot \sqrt{\varepsilon_{r} \mu_{r}}}$.

Below the lower corner frequency $f_{1}$ the system shows differential behavior and above the upper corner frequency $f_{2}$ the system has an integral behavior. The order depends mainly on the number of possible coupling paths into the system. Between both corner frequencies there is the resonance range of the system which has been assumed as constant over the frequency in the following.

For the generation of the test pulses it is necessary to model the previously described coupling behavior. To model the coupling behavior of different electronic systems the lower and upper corner frequency should be variable over a given range. The lower corner frequency is variable between $30 \mathrm{MHz}$ and $1 \mathrm{GHz}$ and the upper one can be changed between $300 \mathrm{MHz}$ and $3 \mathrm{GHz}$. With Eqs. (1) and (2) these frequency ranges include systems with a maximal coupling path length of about $1.5 \mathrm{~m}$ (e.g. the length of a connection cable to a PC system) and a minimal coupling path length of about $10 \mathrm{~cm}$ (size of a typical handheld system). For that investigation an UWB-pulse-generator in combination with different

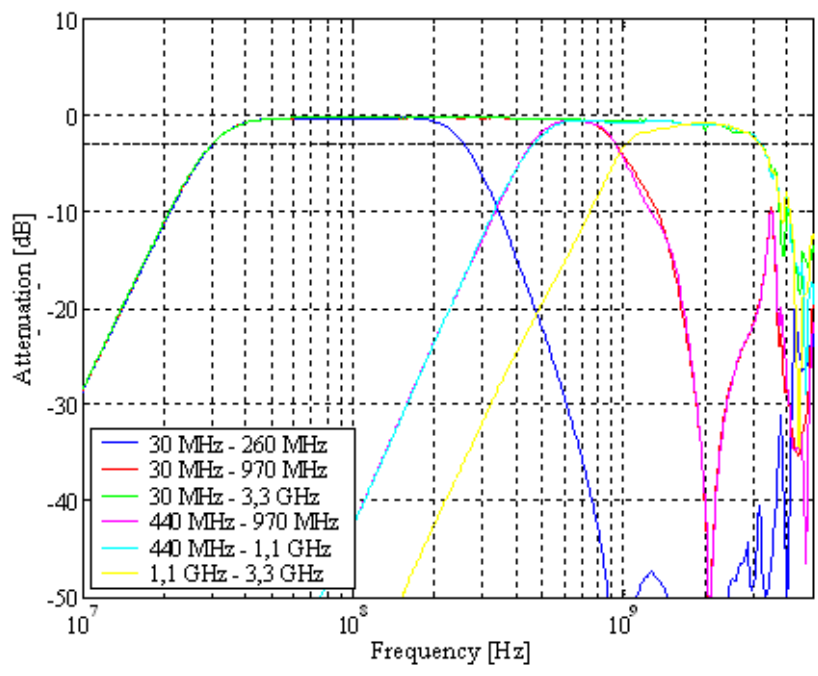

Fig. 2. Measured transfer functions in frequency domain of the realized linear filters to model the coupling behavior of complex electronic systems.

developed high- and lowpass filters has been used to generate the wanted test pulses. Figure 2 shows the transfer functions of the realized filter combinations in frequency domain. The behavior of the combination of high- and lowpass filters is very close to the described behavior of dipole structures. From the different combinations of high- and lowpass filters three representative combinations have been chosen to determine the response behavior of typical nonlinear protection elements by field radiated line coupled UWB-pulses. At first there is the whole frequency range from $30 \mathrm{MHz}$ to $3 \mathrm{GHz}$, second a lower frequency range from $30 \mathrm{MHz}$ to $300 \mathrm{MHz}$ and at last an upper frequency range from $1 \mathrm{GHz}$ to $3 \mathrm{GHz}$. According to Fig. 3 the test pulses have been generated with the chosen filter combinations in connection with an UWBpulse-generator and a load. The generator produces double exponential pulses with a rise time of about $150 \mathrm{ps}$, a pulse width of $2.5 \mathrm{~ns}$ and a voltage amplitude of $12.5 \mathrm{kV}$. The combination of the UWB-pulse-generator and the high- and lowpasses generates the signal which occurs on the line due to coupling effects into system caused by field radiated UWBpulses. To characterize the nonlinear behavior of the protection elements in time domain attenuators have been used in the signal path to provide voltage amplitudes of the test pulses above and below the breakdown voltage of the nonlinear elements. The load models the system which has to be protected. To avoid additional reflections on the line the load has been set to $50 \Omega$. In the presented measurement setup the test pulses have to be measured with a probe which provides a very broad frequency range. For that broadband current probes (e.g. F-2000 by FCC Fischer) or the new broadband high voltage probe picoTEM, developed by the Institute of Measurement Technology and Electromagnetic Compatibility at the Hamburg University of Technology (Weber, 2004) 

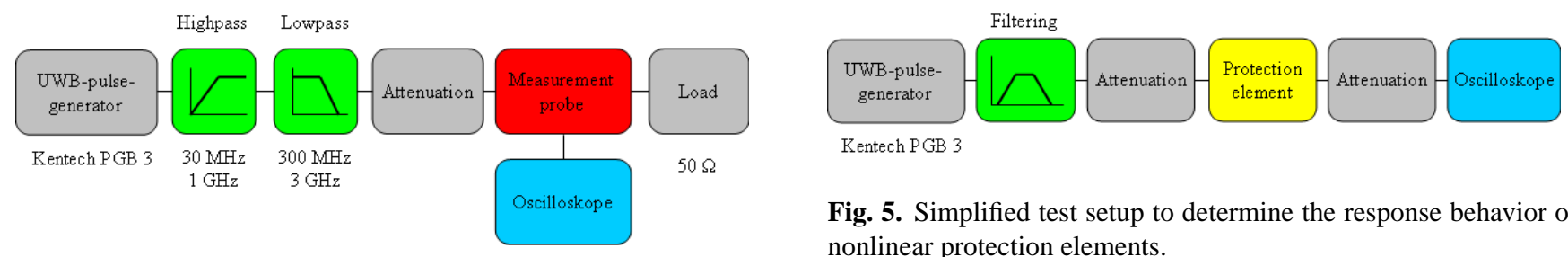

Fig. 3. Measurement setup to determine the time characteristic of the defined test pulses.
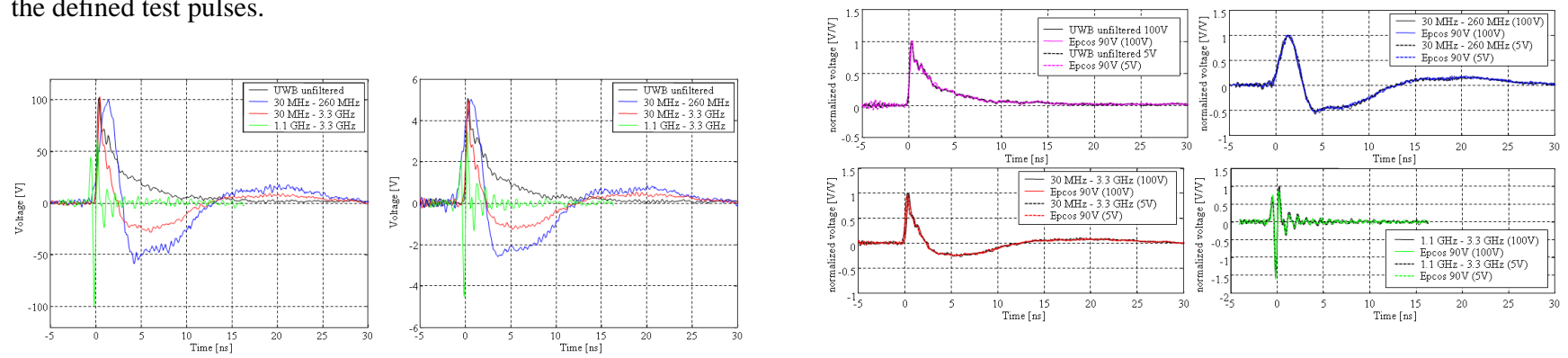

Fig. 4. Time characteristics of the generated test pulses for different coupling bandwidths and different voltage amplitude compared to a direct double exponential UWB-pulse.

can be used. The measured test pulses in time domain are shown in Fig. 4 for different coupling bandwidths and different output voltages. For comparison the direct double exponential UWB-pulse is also shown in this figure.

\section{Investigations of protection elements in time domain}

In the following the response behavior of typical nonlinear protection elements will be determined by impact with the previously defined test pulses. In general typical protection circuits are combinations of spark gaps, varistors and protection diodes. For this investigation often used components have been chosen which have breakdown voltages between the amplitudes of the defined test pulses of $5 \mathrm{~V}$ and $100 \mathrm{~V}$. Additionally a very fast protection diode with a very low parasitic capacitance has been used in the test. The following listed elements have been tested with respect to their response behavior:

- spark gap: EPCOS90V

- varistor: S14K30

- protection diode: BZX85C62

- fast protection diode: GBLC15C

A simplified test setup to measure the response behavior is shown in Fig. 5. The 50- $\Omega$-load has been replaced directly by an oscilloscope. Additional attenuators have been used to protect the input port of the oscilloscope from over voltages. Now the obtained measurement results of the single

Fig. 6. Response behavior of the spark gap EPCOS90V normalized to the voltage amplitude of the respective interfering test pulse.

protection elements will be discussed in detail. For a better comparison of the response behaviors of the elements at different voltage amplitudes at the same pulse shape the measured response behavior has been normalized to the voltage amplitude of the corresponding interfering pulse. If both normalized response behaviors have the same time characteristic the element shows at voltages above and below their breakdown voltage the same behavior. In that case the element has no nonlinear behavior and reacts only due to their parasitic linear elements. If the normalized response behaviors have different pulse shapes the element shows different behaviors at voltages above and below their breakdown voltage. This effect is typical for nonlinear behavior.

\subsection{Spark gaps}

The behavior of spark gaps is determined by the so-called breakdown characteristic which describes the relation between the breakdown voltage of the spark gap and the rise time of the interfering signal. Mostly spark gaps were used in lightning protection applications. Lightnings have rise times in the microsecond range and voltage amplitudes of some $\mathrm{kV}$. The test pulses in this investigation have rise times in the subnanosecond range with maximal amplitudes up to 100 Investigation of the behavior of protection elementsV. Therefore it has not been expected that spark gaps show any reduction behavior on line coupled UWB-pulses. This has been verified by measurements which are shown in Fig. 6 . Almost the normalized response behaviors fit exactly with the normalized interfering test pulses. So no protection effect is provided by spark gaps against field radiated line coupled UWB-pulses. 

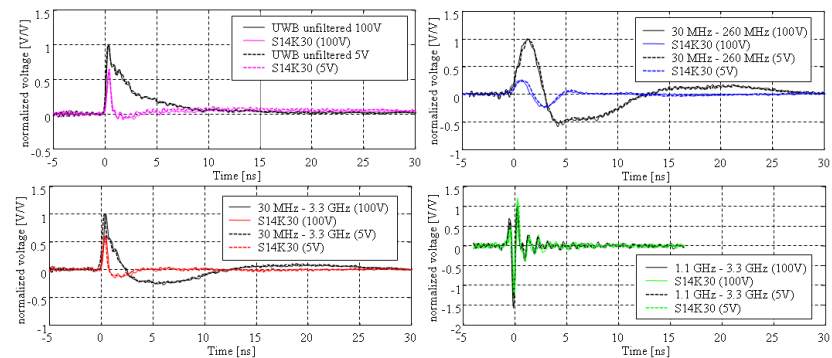

Fig. 7. Response behavior of the varistor S14K30 normalized to the voltage amplitude of the respective interfering test pulse.
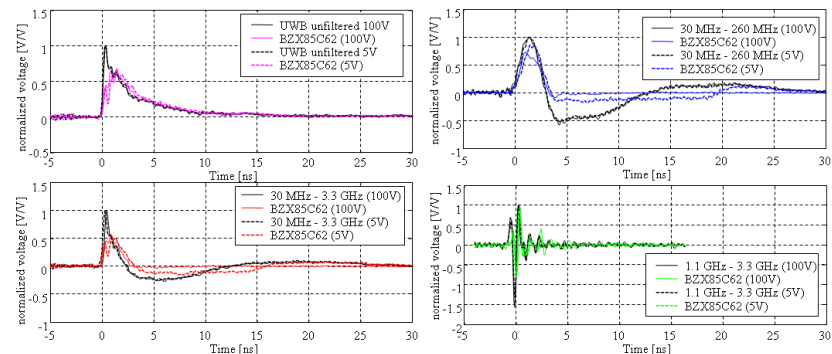

Fig. 8. Response behavior of the protection diode BZX85C62 normalized to the voltage amplitude of the respective interfering test pulse.

\subsection{Varistors}

Just as spark gaps varistors are used as protection elements due to their high energy absorption capability. But the high energy absorption capability is accompanied with a quite large parasitic capacitance of the varistor, whereby varistors only can be used as protection elements in low frequency applications. The measured and normalized response behavior of the chosen varistor is depicted in Fig. 7. Except to the upper frequency coupling range the varistor shows a clear response behavior to the interfering test pulses. However at both test pulses with different voltage amplitudes the normalized response behavior has exact the same pulse shape. As mentioned before in this case the varistor only reacts due to its parasitic linear elements. In fact there is a reduction of the voltage amplitude of the interfering test pulses but this reduction is not systematic and unintentional at both amplitudes of the defined test pulses. That also leads to an influence to a signal with amplitudes below the clamping voltage of the varistor.

\subsection{Protection diodes}

Protection diodes which have in contrast to spark gaps and varistors a much smaller energy absorption capability show again their typical nonlinear behavior at field radiated line coupled UWB-pulses (compare to Fig. 8). At the coupling range from $30 \mathrm{MHz}$ to $300 \mathrm{MHz}$ and from $30 \mathrm{MHz}$ to $3 \mathrm{GHz}$
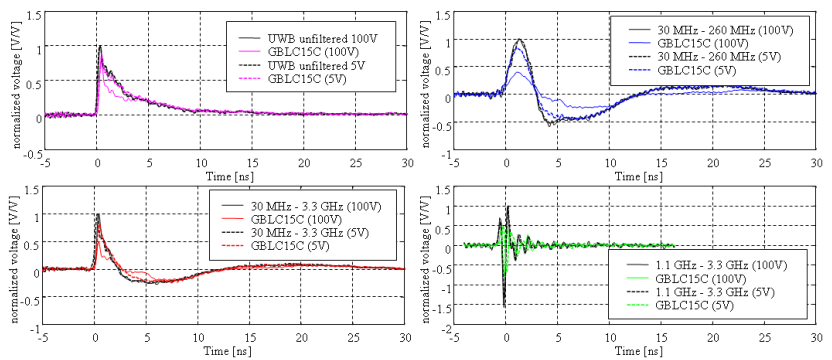

Fig. 9. Response behavior of the low capacitance protection diode GBLC15C normalized to the voltage amplitude of the respective interfering test pulse.

the normalized response behaviors of the protection diode show different pulse shapes which points out the typical nonlinear behavior of the diode. In the upper coupling range from $1 \mathrm{GHz}$ to $3 \mathrm{GHz}$ the tested diode does not show any voltage reduction effects. But at the other two test pulses a reduction of the voltage of the interfering pulses can be observed if the voltage amplitude of the pulses is higher than the breakdown voltage of the diode. Compared to the behavior of the diode at a direct double exponential UWB-pulse the diode shows its typical nonlinear behavior at field radiated line coupled UWB-pulses. However this behavior is highly related to the coupling bandwidth of the system which has to be protected.

\subsection{Protection diodes with an extremely small parasitic capacitance}

Because traditional protection diodes with a small parasitic capacitance have shown better behavior concerning field radiated line coupled UWB-pulses special protection diodes have been tested with an extremely small parasitic capacitance. The normalized measurements are shown in Fig. 9. In contrast to normal protection diodes this diode shows also a voltage limitation of the interfering test pulse at the highest coupling range from $1 \mathrm{GHz}$ to $3 \mathrm{GHz}$ and at the direct double exponential UWB-pulse. At each coupling range the diode shows the typical nonlinear behavior. At voltages above the breakdown voltage of the diode the voltage has been reduced and at voltages smaller that the breakdown voltage of the diode the signal has not been influenced significantly. With these kinds of fast protection diodes a sufficient protection element is available against field radiated line coupled UWBpulses at different coupling bandwidths as well as against direct coupled double exponential UWB-pulses.

\section{Conclusions}

This investigation determines the response behavior of typical nonlinear protection elements concerning interfering signals which are caused by coupled field radiated UWB-pulses 
into complex electronic systems. For that a possibility was presented to generate field radiated line coupled UWB-pulses depending on the coupling bandwidth of the system which has to be protected. With different generated test pulses typical protection elements such as spark gaps, varistors and protection diodes have been tested. But also special protection diodes with extremely small parasitic capacitances have been used in this test. It could be observed that spark gaps did not show any effects at the defined test pulses. The tested varistor has shown a clear reduction of the interfering test pulses but the observed effect was not of nonlinear nature but only caused by the quite high parasitic linear elements of the varistor. Normal protection diodes have shown typical nonlinear behavior again in case of test pulses with lower frequency parts. At the test pulses with frequencies in the upper range the normal diodes also have been react mainly due to their parasitic linear elements. But special protection diodes with an extremely small parasitic capacitance have shown a very good response behavior at all kinds of test pulses so that they can be used against all kinds of UWB-interferences. Concluding it can be said that also field radiated line coupled UWB-pulses can undermine traditional protection circuits. This is mainly related to the coupled frequency parts of the field radiated UWB-pulse into the system. However with special low capacitance diodes the design of sufficient protection circuits should be possible.

\section{References}

Baum, C. E.: From the Electromagnetic Pulse to High-Power Electromagnetics, Proc. of the IEEE, USA, 80, 1992.

Krzikalla, R. and ter Haseborg, J. L.: HPEM protection on HF transmission lines, Kleinheubacher Tagung, Miltenberg, 2003.

Nitsch, D.: Die Wirkung eingekoppelter ultrabreitbandiger elektromagnetischer Impulse auf komplexe elektronische Systeme, Dissertation, Univ. of Technology Hamburg-Harburg, 2005.

Sabath, F.: UWB - Antenne für verbringbare Wirksysteme, Wehrtechnisches Symposium, Mannheim, 2002.

Weber, T.: Messverfahren und Schutzmaßnahmen für Elektromagnetische Pulse im UWB - Bereich, Dissertation, Univ. of Technology Hamburg-Harburg, 2004. 